\title{
Jupiter's post-impact atmospheric thermal response
}

\author{
By B ARNEY J. CONRATH
}

\author{
Laboratory for Extraterrestrial Physics, NASA Goddard Space Flight Center, Greenbelt, MD \\ 20771, USA
}

\begin{abstract}
Measurements of thermal emission in spectral regions, ranging from the near-infrared to $\mathrm{mm}$ wavelengths provide information on the atmospheric thermal structure over impact sites from $\mu$ bar levels in the upper stratosphere down to the upper troposphere. Systematic time series of observations relevant to this entire height range over individual spots do not exist. However, by piecing together information at different times from various spots, it is possible to obtain a provisional, semi-quantitative picture of the behavior of the thermal structure over a typical impact site. Immediately after fall-back of the ejecta plume, the upper stratosphere is heated to $\sim 600-1300 \mathrm{~K}$ above ambient temperature. The amplitude of the temperature perturbation diminishes with increasing depth in the atmosphere, but even in the upper troposphere a temperature increase of a few kelvins is observed. Initially, the upper stratosphere cools very rapidly with time scales of tens of minutes, presumably the result of strong radiative cooling associated with the high temperatures. After the initial cooling, all levels continue to cool at slower rates with time scales of a few days; however, this is still very rapid compared to radiative cooling of the ambient atmosphere. Enhancements in infrared opacity necessary to produce the cooling radiatively do not appear to be viable, suggesting that dynamical effects may play a dominant role. Possible mechanisms include horizontal mixing with the ambient atmosphere and adiabatic cooling produced by upward motion associated with an anticyclonic vortex. Many questions remain concerning the thermal structure above the impact sites; these are being addressed through ongoing data analysis and modeling efforts.
\end{abstract}

\section{Introduction}

The impacting fragments of comet $\mathrm{P} /$ Shoemaker-Levy 9 produced significant local perturbations to Jupiter's atmospheric thermal structure. Detailed study of the behavior of the temperature structure of the impact sites can provide information on the radiative and dynamical properties of these sites and may ultimately prove to be diagnostic of the ambient state of Jupiter's upper troposphere and stratosphere.

In this review, we attempt to synthesize a picture of the spatial and temporal behavior of a typical impact site by examining selected observations relevant to the temperature structure. Ideally, one would like to have a time series of observations of a particular site covering a broad height range. Unfortunately, such a systematic set of measurements does not exist because of many practical considerations including observational constraints and the fact that as the sequence of impacts progressed, many sites overlapped and merged with other sites. Therefore, it is necessary to piece together data from various spots at various times, obtained with a variety of observational techniques.

In the following Section, we first review selected observations relevant to the thermal structure of the various impact sites and their ambient surroundings. In Section 3, we attempt to combine information based on these observations to reconstruct the behavior of thermal structure associated with a "generic spot" as a function of atmospheric pressure level and time after impact. The possible atmospheric radiative and dynamic effects implied by this behavior are considered in Section 4. Finally, the present status of our understanding and some remaining questions are summarized in Section 5. 


\section{Observations}

In this section, we summarize selected observations that are relevant to achieving an understanding of the behavior of the atmospheric thermal structure from the time of fall-back of the ejecta plume until several weeks following impact. This discussion is necessarily based primarily on preliminary reports by various groups of observers. Undoubtedly, an improved understanding of the thermal behavior will emerge in the future as detailed intercomparisons of the data sets are made, and more refined modeling is carried out. We will first examine observations that provide information on atmospheric temperature at and immediately following fall-back. Then we will discuss observations taken during the hours and days following the impacts that are relevant to the thermal behavior over a range of atmospheric levels from the upper stratosphere to the troposphere.

\subsection{Temperatures immediately after fall-back}

Observations in the near-infrared of several sites show evidence of major increases in stratospheric temperatures. Shortly after the impact of fragment H, Encrenaz et al. (1995a; 1995b), using the IRSPEC imaging spectrometer on the New Technology Telescope of the European Southern Observatory in Chile, observed strong brightening in the $3.5 \mu \mathrm{m}$ spectral region. This is a region of strong methane absorption, and, consequently, is usually quite dark under normal Jovian conditions. The solar flux is absorbed with little backscattered radiation present, and at nominal stratospheric temperatures, thermal emission is very small. On this basis, the observed post-impact brightening has been interpreted as thermal emission from methane resulting from an enhanced upper stratospheric temperature. Examples of two of the spectra, the first taken at maximum brightening 14 minutes after impact and the second approximately 6 minutes later, are shown in Fig. 1. By fitting parameterized models to the measurements, Encrenaz et al. infer a temperature of $750 \pm 100 \mathrm{~K}$ near the 0.01 mbar level at the time of maximum emission. By the time of the spectrum shown in Fig. 1b, the temperature had decreased to about $640 \mathrm{~K}$, consistent with a very rapid initial cooling.

Maillard et al. (1995) obtained measurements in the 1.6 to $4.7 \mu \mathrm{m}$ spectral region using a Fourier Transform Spectrometer at the Canada-France-Hawaii Telescope. Approximately 10 minutes after the impact of the $\mathrm{C}$ fragment, they observed strong methane emission consistent with temperatures between 750 and $1500 \mathrm{~K}$ at levels between 0.1 and 0.01 mbar. They also observed CO emission from the L site at $4.7 \mu \mathrm{m} 4.5$ hours after the impact. Model calculations suggest a temperature of $274 \pm 10 \mathrm{~K}$. This is found to correspond to approximately the $2 \mu$ bar level if the $\mathrm{CO}$ mixing ratio profile derived by Lellouch et al. (1995) from millimeter observations of the $G$ site is assumed. CO emission from the L site at $4.7 \mu \mathrm{m}$ was also observed by Brooke et al. (1995) about 4 hours after impact using the CGS4 spectrometer on the United Kingdom Infrared Telescope (UKIRT). Knacke et al. (1995), also using the UKIRT, infer initial temperatures near $2000 \mathrm{~K}$ from $2.2-2.4 \mu \mathrm{m}$ spectroscopy of the R event.

Crisp \& Meadows (1995) obtained measurements with the Infrared Imaging Spectrometer on the Anglo-Australian Telescope that indicate strong near-infrared CO line emission from several sites, apparently associated with fall-back events. Rotational temperatures in excess of $2000 \mathrm{~K}$ were inferred; however, the emission decayed rapidly, and could no longer be detected by about 30 minutes after impact.

Emission in the $3.4 \mu \mathrm{m}$ region has been directly observed by NIMS on Galileo during the $\mathrm{G}$ and $\mathrm{R}$ events (Carlson et. al. 1995). Under the assumption that the emission originates from a continuum of particulates, Carlson et al. infer a temperature immediately after fall-back of the ejecta of $1000 \mathrm{~K}$ or less. 

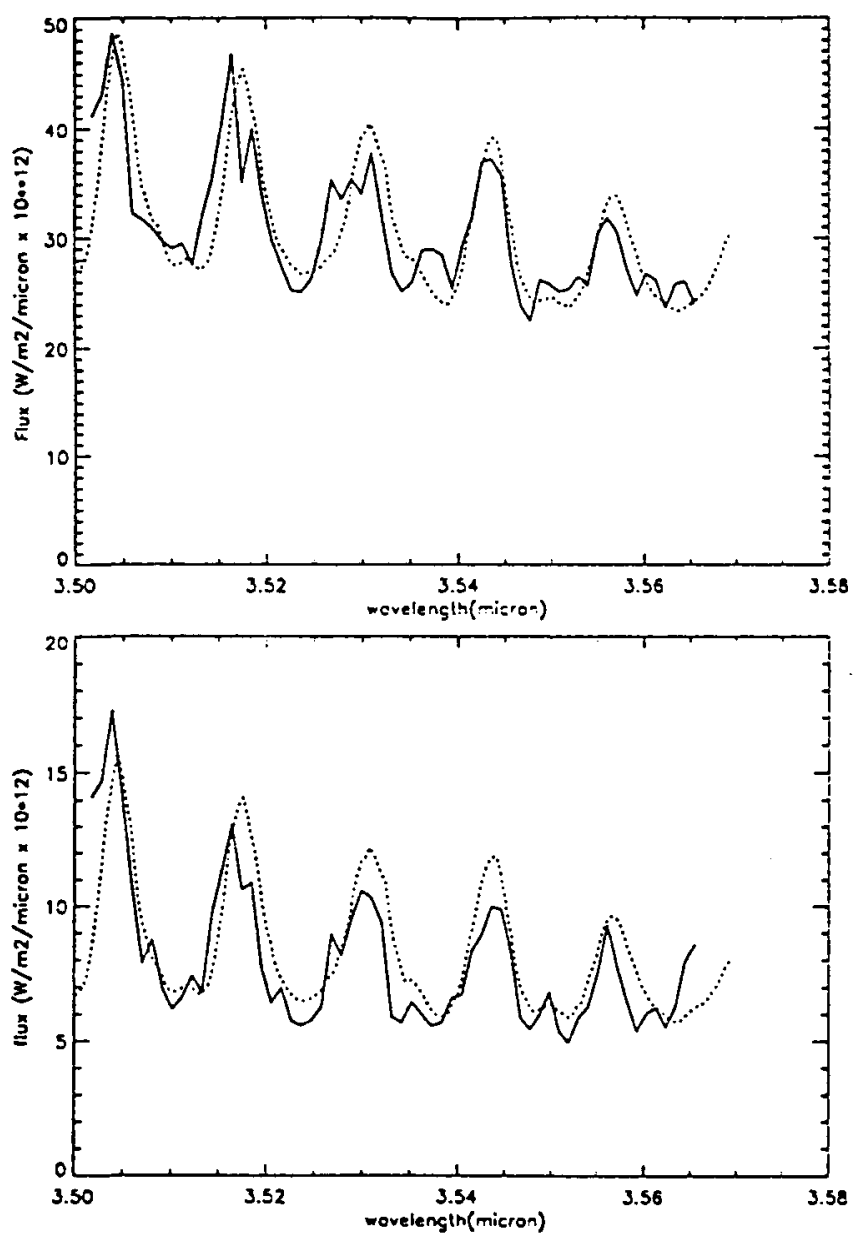

FIGURE 1. Spectra obtained with the IRSPEC imaging spectrometer at the European Southern Observatory. Measurements are shown as solid curves and model fits as broken lines. The spectrum in the upper panel was acquired 14 minutes after impact $\mathrm{H}$, and the model fit was obtained assuming a temperature of $750 \mathrm{~K}$ near $0.01 \mathrm{mbar}$. The lower panel shows a spectrum taken 6 minutes later, along with a model spectrum calculated assuming a temperature of $640 \mathrm{~K}$ near $0.01 \mathrm{mbar}$. Note the change in flux scale between the upper and lower panels. (From Encranez et al. 1995.)

Observing with the MIRAC2 mid-infrared array camera on the IRTF, Friedson et al. (1995) found enhanced emission at $7.85,10.3$, and $12.2 \mu \mathrm{m}$ in the time period immediately following the impact of fragment $R$. From these data, it is not possible to uniquely infer both the angular extent of the source and the temperature. However, for a source $1900 \mathrm{~km}$ in diameter, Freidson et al. estimate a temperature of at least $1350 \mathrm{~K}$, while if the source were a factor of two larger, the inferred temperature would be $800 \mathrm{~K}$. In the nominal Jovian atmosphere, these measurements would be indicative of temperatures in approximately the 1-10 mbar region. The presence of possible additional gaseous and particulate opacity associated with the impact would tend to move the region of sensitivity toward lower pressures.

The measurements summarized above pertain to several different spots, are in some cases from different spectral regions, and were interpreted under various modeling as- 

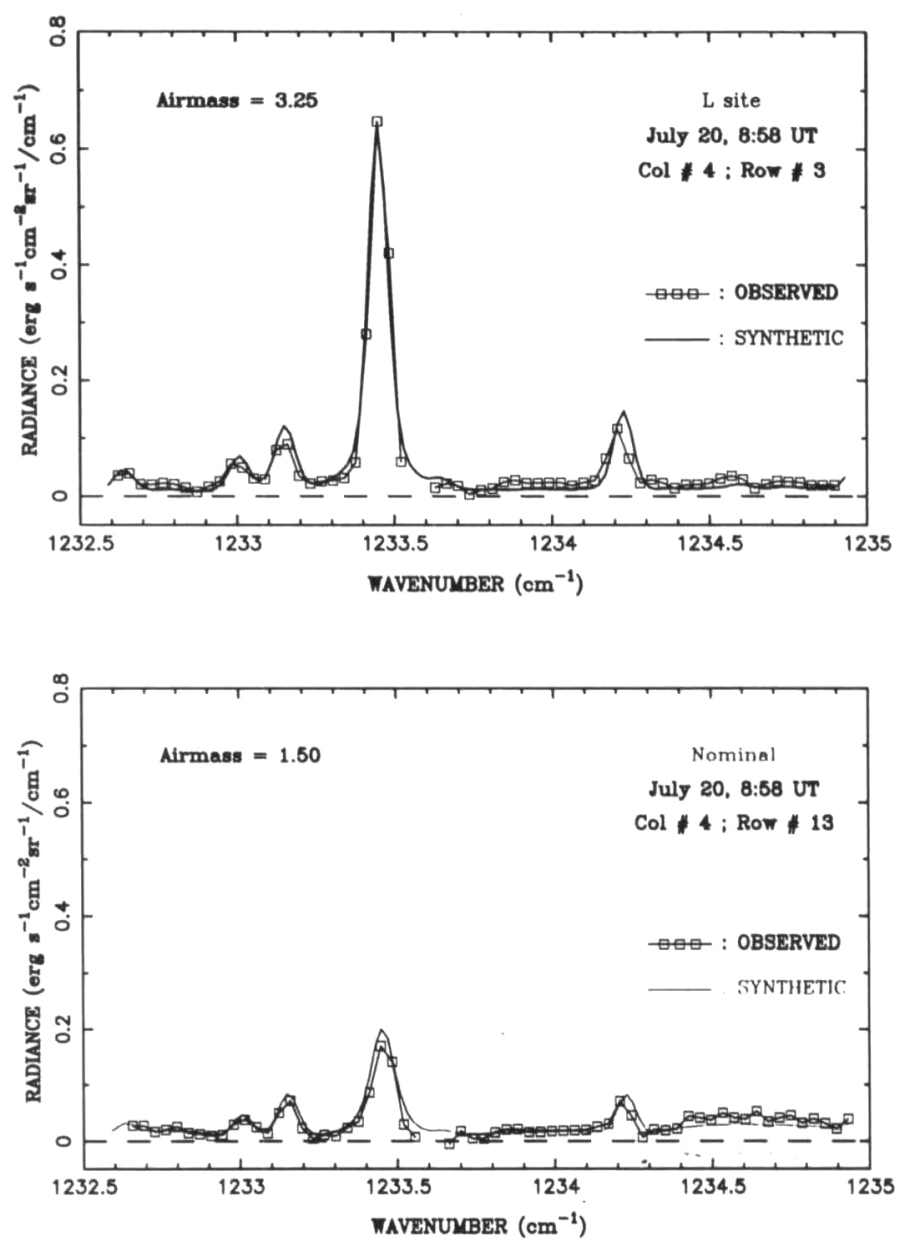

FIGURE 2. Irshell spectra obtained from the $L$ impact site (upper panel) and an adjacent area (lower panel). Both observed and synthetic spectra are shown as indicated. A strong methane absorption lines is seen at $1233.455 \mathrm{~cm}^{-1}$ with weak lines at 1233.147 and $1234.226 \mathrm{~cm}^{-1}$. The measurements were acquired approximately 11 hours after impact. (From Bézard et al. 1995.)

sumptions so it is difficult to synthesize a completely coherent picture at this time. However, it does appear that the upper stratospheric temperatures were strongly perturbed by the fall-back or "splash", perhaps ranging from about 700 to $2000 \mathrm{~K}$ in the region between approximately $1 \mathrm{mbar}$ and a few $\mu$ bars. The heating was followed by a very rapid cooling with temperatures probably dropping over $100 \mathrm{~K}$ in a matter of minutes.

\subsection{Irshell observations}

Next, measurements relevant to the behavior of temperatures following the initial cooling phase will be considered. We shall begin in the upper stratosphere and work our way downward.

Measurements with the Irshell, a mid-infrared high spectral resolution imaging spectrometer, have been used by Bézard et al. (1995) to infer information on the atmospheric temperatures above about the 0.1 mbar level. Spectra obtained from the $\mathrm{L}$ site and an adjacent region approximately 11 hours after impact are shown in Fig. 2. Within the narrow spectral region covered there is both a strong and a weak methane line. The strong 


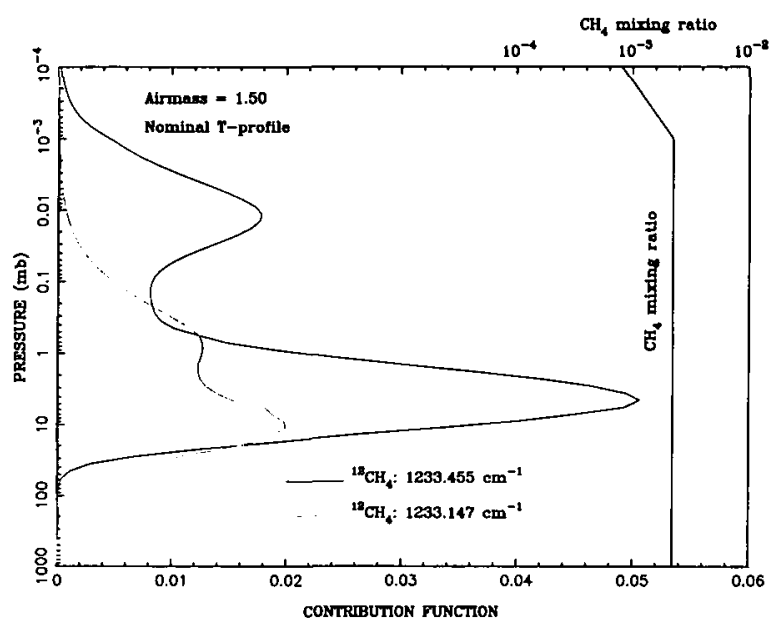

FIgure 3. Contribution functions for a strong and a weak methane absorption line. The relative contributions of each atmospheric level to the radiance at the top of the atmosphere are shown. The radiance from the strong line at $1233.455 \mathrm{~cm}^{-1}$ contains contributions from both the upper and lower stratosphere, while that from the weak line at $1233.147 \mathrm{~cm}^{-1}$ includes contributions primarily from the lower stratosphere. The methane profile used in the models is shown in the right-hand side of the figure. (From Bézard et al. 1995.)

line shows a much more intense emission on the site than off, while the enhancement of emission from the weak line on the site is significantly less. By combining measurements from these two lines, Bézard et al. were able to infer information on the behavior of the temperature perturbation as a function of pressure level. The contribution functions for these two lines are shown in Fig. 3.

The strong line emission includes contributions from both the lower and upper stratosphere, while for the weaker line, the contributions are mostly from the lower stratosphere. This permits a crude separation of information on the temperature perturbation of the upper stratosphere from that of the lower stratosphere. Model temperature structures that provide good fits to the spectra are shown in Fig. 4. Because of the limited information, the details of the inferred structure are highly non-unique and two families of solutions are shown. However, the results clearly constrain the primary temperature perturbation to lie mainly above the 1 -mbar level. The maximum temperatures permitted by the weak line observations are also shown. Results were also obtained by Bézard et al. for the $\mathrm{K}$ site 23 hours after impact showing a weaker perturbation. The measurements appear to be consistent with a continued cooling of the sites after the fall-back, but at a much lower rate than that initially observed immediately after the splash.

\subsection{IRAM Observations}

Strong emission by $\mathrm{CO}$ in the millimeter wavelength region was observed with the IRAM telescope by Lellouch et al. (1995). The spots were not spatially resolved because of the relatively large beam size of the telescope, and most of the observations pertain to the G-Q-R-S complex. The emission was observed to decay rapidly over several days time. In the interpretation of these data, it is not possible to uniquely determine both the $\mathrm{CO}$ abundance and atmospheric temperature. Based on considerations of the $\mathrm{CO}$ chemistry, it was assumed that the $\mathrm{CO}$ abundance remained constant over the period of the observations. Under this assumption, it was found that the temporal evolution of thermal structure could be modeled as shown in Fig. 5. The temperature profile labeled 


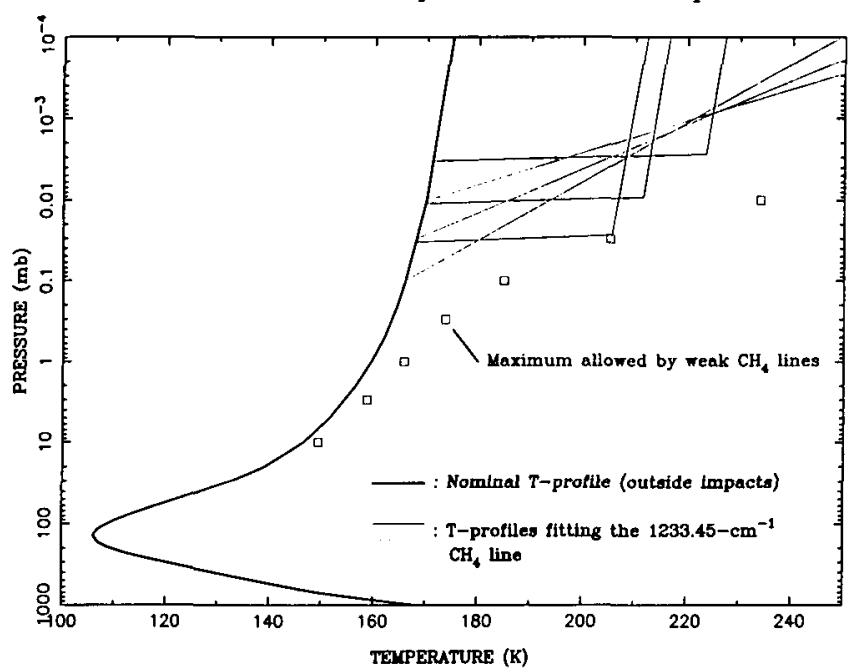

FIGURE 4. Model temperature profiles for the $L$ impact site and adjacent region approximately 11 hours after impact. The heavy solid curve is the nominal profile that fits the spectrum from the adjacent region. The thin curves represent families of profiles that all fit the spectrum from the impact site equally well. The square symbols represent the highest temperatures permitted by the weak methane lines. (From Bézard et al. 1995.)

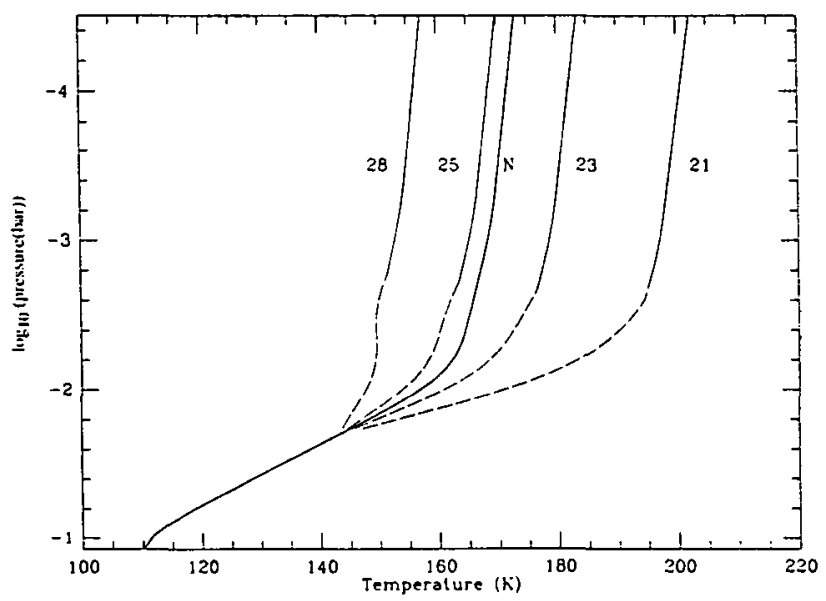

FIGURE 5. Model temperature profiles used to fit measurements obtained with the IRAM telescope. The curves are labeled with the July 1994 date on which they were taken, and the curve labeled $\mathrm{N}$ is taken to be the nominal profile. The measurements are sensitive to levels only above about the 1 mbar level. (From Lellouch et al. 1995.)

$\mathrm{N}$ was taken as nominal. The measurements are sensitive primarily to the atmospheric region above approximately $1 \mathrm{mbar}$, and little detailed vertical structure information is available. The numbered profiles correspond to the dates in July 1994 on which the observations were made. A very rapid cooling is noted with a characteristic time scale of a few days. Remarkably, the upper stratosphere appears to cool significantly below the assumed nominal temperature.

\subsection{SpectroCam-10 observations}

Observations of impact sites were made in the middle infrared by Nicholson et al. (1995a; 1995b) using SpectroCam-10, an imaging array spectrometer on the 5-meter Hale tele- 


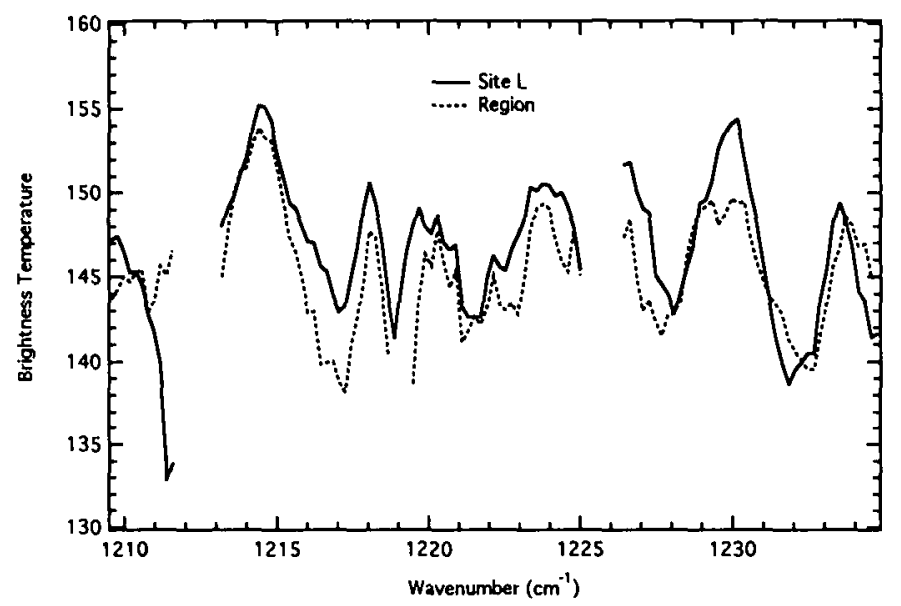

FIgURE 6. Measurements obtained with SpectroCam-10 on the 5-meter Hale telescope at Palomar. The solid curve is a spectrum taken on the $L$ site approximately 4 hours after impact, and the broken curve is a spectrum from an adjacent region. The primary absorber in this region is methane. (From Conrath et al. 1995.)

scope at Palomar. Both low resolution $\left(10 \mathrm{~cm}^{-1}\right)$ spectra in the 8-12 $\mu \mathrm{m}$ region and high resolution $\left(0.5 \mathrm{~cm}^{-1}\right)$ spectra in selected narrow intervals were acquired. An example of high resolution spectra between 1210 and $1240 \mathrm{~cm}^{-1}$ of the $\mathrm{L}$ site and an adjacent region is shown in Fig. 6. This spectral region is dominated by methane emission from the stratosphere and is sensitive to temperature in a broad layer between approximately 1 and 10 mbar. Comparison of the two spectra show enhanced emission over the $L$ site compared to the adjacent region. These measurements were combined with low resolution spectra to retrieve estimates of temperature structure in the 1-10 mbar region and near the tropopause and upper troposphere (Conrath et al. 1995). The results are shown in Fig. 7. Although extremely crude in terms of vertical structure information, the results indicate a temperature perturbation above the $10 \mathrm{mbar}$ level of about $5 \mathrm{~K}$. There may be a slight warming in the upper troposphere, but the apparent warming at deeper levels is an artifact of the retrieval since the measurements do not contain information on these levels. The results were obtained about 4 hours after the impact.

\subsection{MIRAC2 observations}

Data obtained with MIRAC2, an imaging middle infrared array camera at the IRTF, have been used to infer information on the thermal structure of several impact sites (Orton et al. 1995a). Measurements in a spectral interval centered at $7.85 \mu \mathrm{m}$ are sensitive to a relatively thick layer centered near the 10 mbar level. From these images, temperature perturbations over the site relative to the surrounding regions were inferred. Site $E$ on July 18 was found to have a perturbation of about $1.5 \mathrm{~K}$ which decreased to less than $0.5 \mathrm{~K}$ in about 3 days. A thermal perturbation of $2 \mathrm{~K}$ was observed over site $\mathrm{L}$ on July 20, but had decayed to about $1 \mathrm{~K} 19$ hours later, and was no longer detectable when the site was observed again on July 28. Observations of the Q1+R site 10 hours after the $\mathrm{R}$ impact indicated an temperature enhancement relative to the surrounding region of $3-4 \mathrm{~K}$.

Orton et al. (1995b) have used images at 13.0, 17.8, 20.2, and $20.8 \mu \mathrm{m}$ to retrieve information on thermal perturbations in the upper troposphere. Temperature retrievals from these data are shown in Fig. 8 for site $\mathrm{K}$ on July 28 and sites G and L on July 21. 

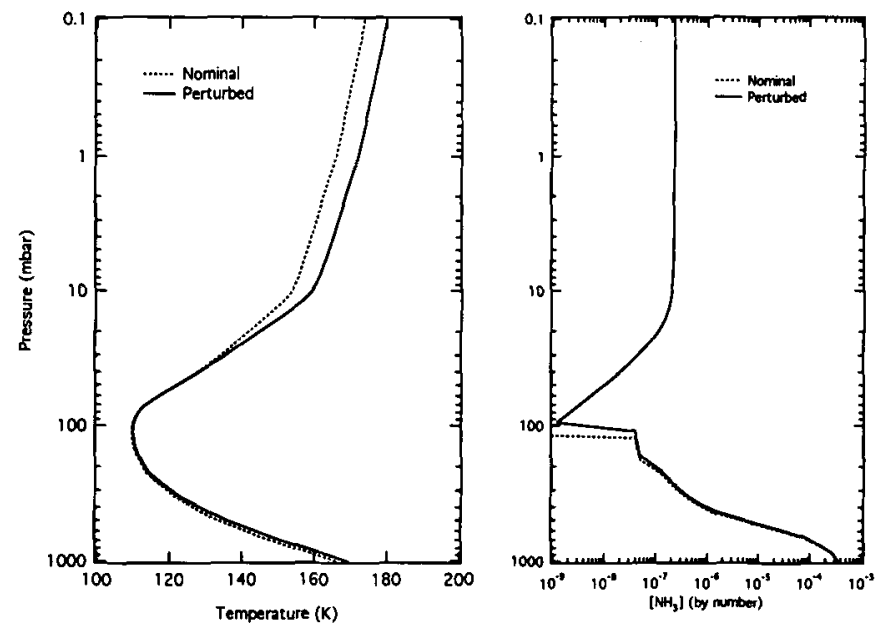

Figure 7. Profiles of temperature (left panel) and ammonia (right panel) retrieved from SpectroCam-10 measurements. The broken curves labeled nominal are for the region adjacent to the impact site and the solid curves are from measurements on impact site $L$. The retrievals are meaningful only in the 1-10 mbar region and in the upper troposphere. (From Conrath et al. 1995.)

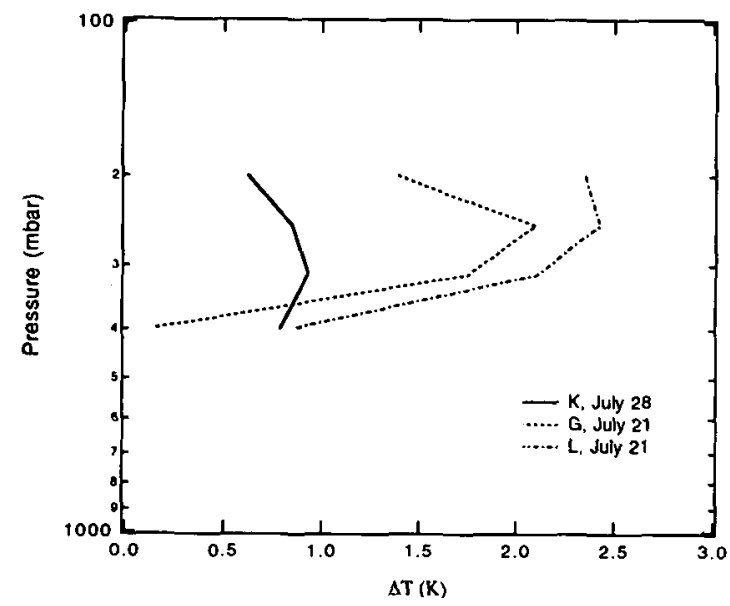

FIGURE 8. Tropospheric temperature retrievals obtained from MIRAC2 measurements. The retrievals are expressed in terms of a temperature perturbation over a site relative to an adjacent region. Results for the the K, G, and $L$ sites are shown. The measurements used for the $K$ site were taken July 28 and those for the G and L sites were obtained on July 21. (From Orton et al. 1995.)

Temperature perturbations are shown relative to surrounding regions. They appear to be confined to the atmospheric levels above approximately 400 mbar.

\section{Synthesis of results}

In an attempt to organize the thermal structure data, we schematically show the various results discussed above in Fig. 9. The temperature perturbations are shown as temperature differences $\Delta T$ relative to the nominal jovian atmosphere. In most cases the nominal thermal structure is taken to be that in unperturbed regions near the impact 


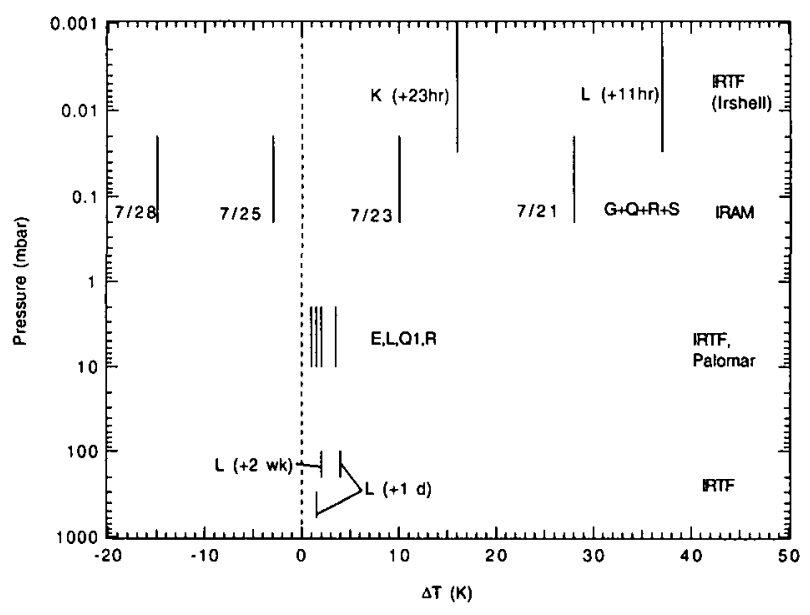

FIgURE 9. Schematic summary of temperature inferences. The approximate vertical range of sensitivity of each determination is shown as a vertical line. The results are expressed as temperature perturbations $\Delta T$ over the sites relative to the ambient atmosphere. The site to which each result pertains is indicated along with either the date of the observation or time after impact. The facility used is indicated on the right of the figure.

sites. The approximate pressure ranges to which the results apply are shown. Temporal variations are indicated where available. The temperature perturbations of 600 to $1000 \mathrm{~K}$ observed immediately after fall-back of the impact-generated plumes are not included in this figure.

Unfortunately, a time-history of the thermal perturbation associated with any one particular spot at all relevant atmospheric levels does not exist. In the absence of such direct information, we have attempted to combine the available measurements from several spots shown in Fig. 9, and postulate the thermal behavior of a "generic" spot as shown in Fig. 10. However, the over-all picture that seems to emerge is one of very strong heating of the upper stratosphere by the impacting ejecta plume as it falls back into the atmosphere. This is followed by a very rapid drop in temperature with a characteristic time scale of the order of minutes. Presumably this is due to strong radiative cooling associated with the very high initial temperatures. With decreasing temperatures the cooling rate decreases; several hours after impact, the characteristic cooling time in the upper stratosphere is of the order of 2-3 days. It is this phase of the temporal evolution that is depicted schematically in Fig. 10. While the detailed vertical structure of the temperature perturbation has not been well established, it does decrease significantly with increasing pressure in the lower stratosphere. The indicated "overshoot" in cooling with the temperature near 0.1 mbar dropping below the nominal value is based on the IRAM observations and their model-dependent interpretation.

The behavior of the temperature structure between 10 and 100 mbar, as indicated in the figure, is largely an interpolation between higher levels and the troposphere since none of the available measurements is very sensitive to this portion of the atmosphere. The upper troposphere appears to cool somewhat more slowly than the stratosphere. The cause of the tropospheric temperature perturbation is not well understood at the present time. Possibilities include local heating at the time of impact, downward penetration of heating following ejecta plume fall-back, or a buoyant plume rising adiabatically as the result of impact perturbations at deeper levels. Further investigations of the horizontal 


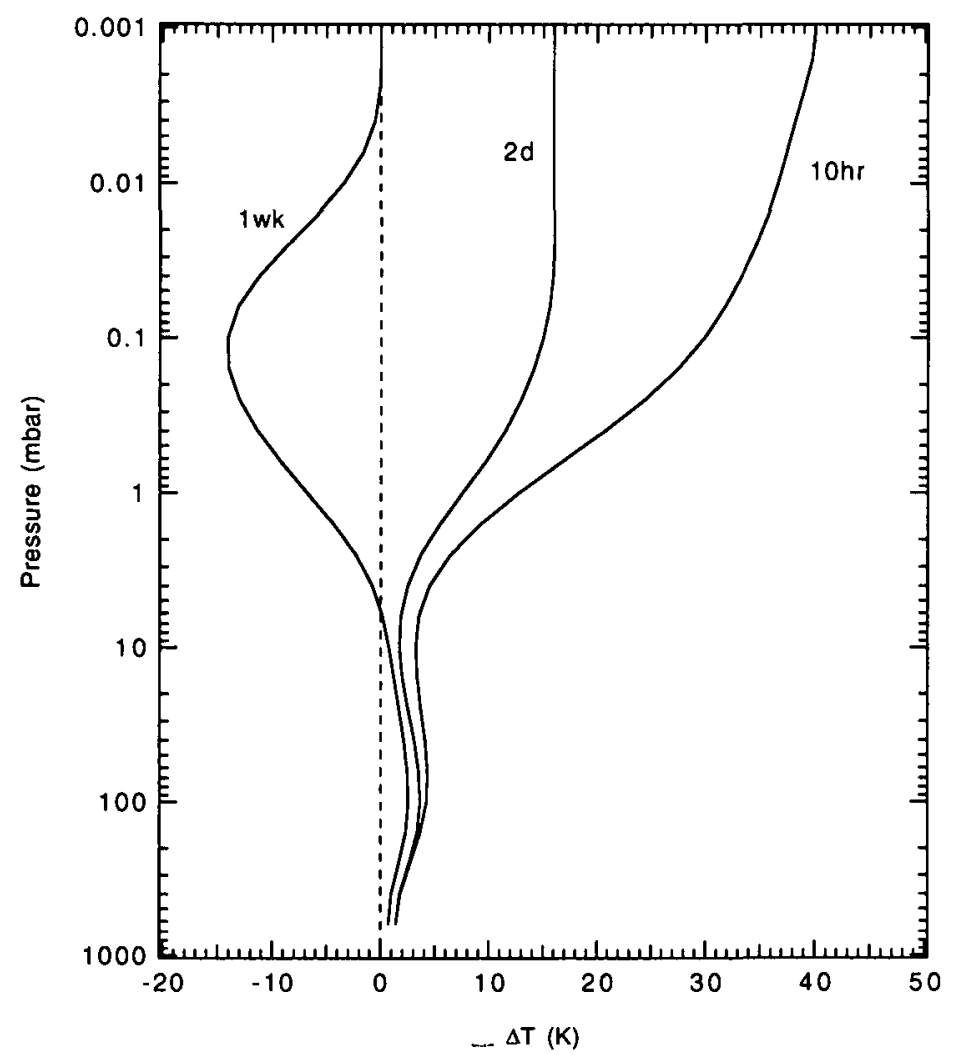

FIgURE 10. Thermal behavior of a "generic" spot. This cartoon of the vertical structure and temporal behavior of the thermal perturbation over a spot relative to the ambient atmosphere is based on the results summarized in Fig. 9. The curve labels indicate time after impact.

spatial extent of the tropospheric perturbations relative to those in the stratosphere may lead to a better understanding of this phenomenon.

\section{Radiative and dynamic effects}

The behavior of the thermal structure over the impact sites raises questions concerning radiative and dynamic processes that may be associated with the sites and their temporal evolution. The very rapid initial cooling rates in the upper stratosphere can be understood, at least qualitatively, in terms of the initial very high temperatures. However, several hours after impact when the upper stratospheric temperatures have returned to within a few tens of $\mathrm{K}$ of equilibrium, apparent cooling times of a few days are observed, which are long compared to those of the initial cooling, but are still short compared to those associated with the ambient jovian atmosphere. This appears to be true at all levels, including the more slowly cooling upper tropsophere.

An example of a radiative time constant calculated for the ambient jovian atmosphere is shown as a function of pressure level in Fig. 11 (Flasar, 1989). Although the time constant is not shown for pressures less than $10 \mathrm{mbar}$, it will continue to increase with decreasing pressure. From this we see that the ambient radiative time constant ranges from about 600 days near the tropopause to over 1000 days in the upper stratosphere. If the rapid cooling is radiative, then an increase in infrared opacity above ambient is 


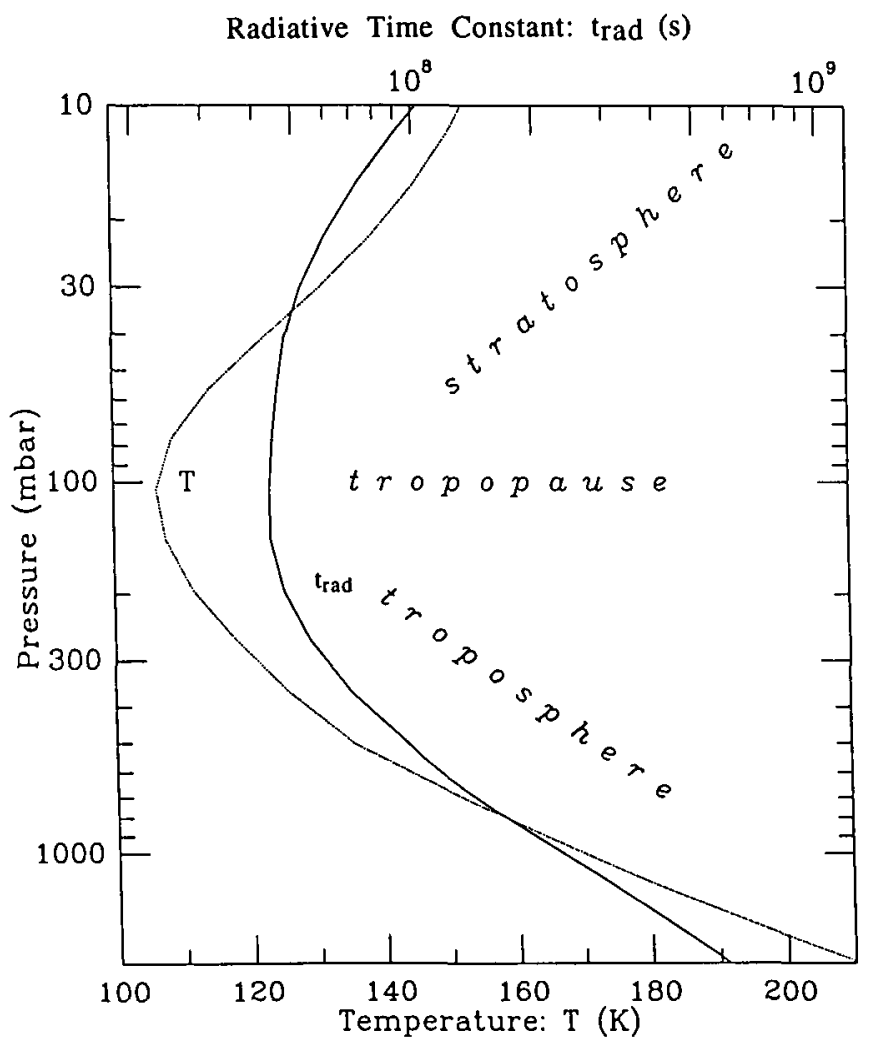

FIguRE 11. Radiative time constant or radiative relaxation time $\left(t_{\text {rad }}\right)$ as a function of atmospheric pressure level for the nominal jovian atmosphere. The temperature profile used to calculate the time constant is labeled $T$. The time constant is expressed in seconds. (From Flasar, 1989.)

required. This could result from either an increased gas opacity or increased particulate opacity.

We can make a rough estimate of the increase in infrared opacity needed to yield the necessary radiative time constant by considering a gray atmospheric emitting layer at temperature $T_{0}$ with a pressure difference across the layer $\Delta p$ and an optical thickness $\Delta \tau$. The radiative time constant is then given approximately by

$$
t_{\text {rad }}^{-1} \sim \frac{4 g \sigma T_{0}^{3}}{c_{p}} \frac{\Delta \tau}{\Delta p}
$$

where $g$ is gravitational acceleration, $\sigma$ is the Stefan-Boltzmann constant and $c_{p}$ is the specific heat of the atmosphere (see for example Andrews et al. 1987). For $p=1$ mbar and $T_{0}=160 \mathrm{~K}$, a radiative time constant $t_{\text {rad }} \sim 1$ day requires a gray optical thickness $\Delta \tau>1$. Such a large additional optical depth from either particulates or enhanced gaseous absorption does not seem to be consistent with mid-infrared spectral measurements (Conrath et al. 1995). If the additional opacity were due to particulates associated with the impact sites, it is likely that they would also produce heating due to absorption of solar energy, and this would partially offset any enhanced infrared cooling, further compounding the problem. Thus, it appears unlikely that radiative cooling alone can account for the observed cooling rates near 10 mbars and deeper. This conclusion is sup- 
ported by detailed, non-gray radiative transfer calculations carried out by Wang et al. (1995).

At higher levels in the atmosphere, smaller optical thicknesses are required to produce the necessary cooling rates. For example, for $p=0.1 \mathrm{mbar}$ and $T_{0}=180 \mathrm{~K}$, a radiative relaxation time of 1 day requires a gray optical thickness of only $\sim 0.05$. This is probably near the upper limit of particulate optical depth in this part of the atmosphere that would be permitted by the mid-infrared spectral measurements.

It appears likely that dynamical effects may be primarily responsible for the rapid decay of the temperature perturbations. Horizontal mixing of the perturbed region with the ambient atmosphere could play a role. A horizontal wind shear of the order several tens of meters per second over a scale of $10^{4} \mathrm{~km}$ might be capable of producing such mixing. Adiabatic cooling associated with upward motion could also contribute. Some spots apparently show some indication of anticyclonic vorticity (Beebe 1995). If such is the case, an upward motion of the central region would be expected with an associated adiabatic cooling given by

$$
\left(\frac{\partial T}{\partial t}\right)_{a d} \sim-w\left(\frac{\partial T}{\partial z}+\frac{g}{c_{p}}\right) .
$$

For a cooling rate $(\partial T / \partial t)_{a d} \sim 1 \mathrm{~K} /$ day, we find a vertical velocity $w \sim 0.5 \mathrm{~cm} \mathrm{~s}^{-1}$. For a spot size of $\sim 10^{4} \mathrm{~km}$ and a depth of the order of one pressure scale height, mass continuity requires a radial outflow of $\sim 2.5 \mathrm{~m} \mathrm{~s}^{-1}\left(250 \mathrm{~km}\right.$ day $\left.{ }^{-1}\right)$. From these considerations, it seems plausible that some combination of dynamic effects is probably responsible for the observed cooling, following the brief very rapid radiative cooling phase associated with the high initial temperatures. This can be confirmed only with more quantitative modeling.

\section{Summary}

Several sets of thermal emission data, acquired during the fragment impacts and in the days immediately following the impacts, have been used to extract information on the perturbed atmospheric temperature structure over the sites. The measurements range from the near-infrared to millimeter wavelengths. Collectively, these measurements yield some information on the atmosphere between a few $\mu$ bars and about 500 mbar. No uniform sets of measurements exist for an individual spot that can provide a complete time history of the thermal behavior at all atmospheric levels. We can only attempt to combine information from various sites at various times to obtain a semi-quantitative picture of the behavior of a "typical" spot.

In the time period immediately following the fall-back of the ejecta-plume, or the socalled splash, temperatures in the upper stratosphere were $\sim 600-1300 \mathrm{~K}$ above ambient. The amplitude of the temperature perturbations decreased with increasing depth in the atmosphere as might be expected if the atmospheric heating is primarily due to the fallback of material. However, temperatures a few Kelvins higher than normal were observed all of the way down into the upper troposphere to about the $500 \mathrm{mbar}$ level. The thermal perturbations in the upper stratosphere initially decayed very rapidly with characteristic time scales of tens of minutes. This rapid change presumably resulted from very strong radiative cooling associated with the high initial temperatures. As the temperatures dropped, the cooling rate also decreased. Several hours after impact, temperatures in the 0.001 to 0.1 mbar region were a few tens of $K$ above ambient, while near 10 mbar, perturbations of only $3-4 \mathrm{~K}$ were observed. Temperatures then continued to decrease in these regions with time scales of the order of days. In the upper troposphere, somewhat 
longer cooling times were noted. However, in all cases the cooling times appear to have been at least two orders of magnitude shorter than estimates of radiative cooling times for the ambient jovian atmosphere.

It is difficult to account for cooling times of the order of days entirely in terms of enhanced radiative cooling resulting from increased infrared opacities. Estimates of the required equivalent gray opacities suggest values so large that they may be inconsistent with middle infrared spectral observations. More detailed radiative transfer calculations support the conclusion that radiative cooling cannot be the primary factor, at least in the lower statosphere and upper troposphere; dynamic effects must therefore be significant. Candidates include horizontal mixing with the surrounding environment and adiabatic cooling, possibly associated with the formation of an anticyclonic vortex. A more detailed examination of the dynamics associated with the sites is needed.

Observations of the thermal structure over the impact sites have raised many questions that remain to be answered. What processes control the vertical structure above a spot? Is the structure diagnostic of spot dynamics? What are the detailed mechanisms that produce rapid stratospheric cooling? What role do particulates play in the structure and evolution of a site? What is responsible for the thermal perturbations in the upper troposphere? On-going data analysis and modeling should provide some answers to these and related questions in the future.

\section{REFERENCES}

Andrews, D. G., Holton, J. R., \& Leovy, C. B. 1987 Middle Atmosphere Dynamics. Academic Press.

BEEBE, R. 1995 Growth and disperison of the Shoemaker-Levy 9 impact features from HST imaging. This volume.

Bézard, B., Griffith, C. A., Kelly, D., Lacy, J., Greathouse, T., \& Orton, G. 1995 Mid-IR high-resolution spectoscopy of the SL9 impact sites: temperature and HCN retrievals. Paper presented at IAU Colloquium 156: The Collision of Comet P/ShoemakerLevy 9 and Jupiter, Baltimore, Maryland, 9-12 May.

Brooke, T. Y., Orton, G. S., Crisp, D., Friedson, A. J., \& BJoraker, G. 1995 Nearinfrared spectroscopy of the Shoemaker-Levy 9 impact sites with UKIRT: CO emission from the $\mathrm{L}$ site. Paper presented at IAU Colloquium 156: The Collision of Comet P/ShoemakerLevy 9 and Jupiter, Baltimore, Maryland, 9-12 May.

Carlson, R. W., Weissman, P. R., Segura, M., Hui, J., Smythe, W. D., Johnson, T., Baines, K. H., Drossart, P., Encrenaz, Th., Leader, F. 'E., \& the NimS Science TEAM 1995 Galileo infrared observations of the Shoemaker-levy 9 G impact fireball: a preliminary report. Geophysical Research Letters 22, 1557-1560.

Conrath, B. J., Gierasch, P. J., Hayward, T., McGhee, C., Nicholson, P. D., \& Van Cleve, J. 1995 Palomar mid-infrared spectroscopic observations of comet ShoemakerLevy 9 impact sites. Paper presented at IAU Colloquium 156: The Collision of Comet P/Shoemaker-Levy 9 and Jupiter, Baltimore, Maryland, 9-12 May.

Crisp, D. \& MEAdows, V. 1995 Near-infrared imaging spectroscopy of the impacts of SL9 fragments C, D, G, K, N, R, V, and W with Jupiter. Paper presented at IAU Colloquium 156: The Collision of Comet $\mathrm{P} /$ Shoemaker-Levy 9 and Jupiter, Baltimore, Maryland, 9-12 May.

Encrenaz, Th., Schulz, R., Stuwe, J. A., Wiedemann, G., Drossart, P. \& Crovisier, J. 1995 Near-ir spectroscopy of Jupiter at the time of comet Shoemaker-Levy 9 impacts: emissions of $\mathrm{CH}_{4}, \mathrm{H}_{3}^{+}$, and $\mathrm{H}_{2}$. Geophysical Research Letters 22, 1577-1580.

Flasar, F.M. 1986 In Time Variable Phenomena in the Jovian System (eds. M. Belton, G. Hunt, \& R. West) Spec. Publ. 494, National Aeronautics and Space Administration, Washington, DC, p. 324 . 
Friedson, A. J., Hoffmann, W. F., Goguen, J. D., Deutsch, L. K., Orton, G. S., Hora, J. L., Dayal, A., Spitale, J. N., Wells, W. K., \& Fazio, G. G. 1995 Thermal infrared lightcurves of the impact of comet Shoemaker-Levy 9 fragment R. Geophysical Research Letters 22 1569-1572.

Knacke, R. F., Fajardo-Acosta, Geballe, T. R., \& Noll, K. S. 1995 Near-infrared spectroscopy of the $R$ impact site of comet Shoemaker-Levy 9. Paper presented at IAU Colloquium 156: The Collision of Comet P/Shoemaker-Levy 9 and Jupiter, Baltimore, Maryland, 9-12 May.

Lellouch, E., Paubert, G., Moreno, R., Festou, M. 'C., Marten, A., Despois, D., Strobel, D. F., \& Sievers, A. 1995 Chemical and thermal effects in Jupiter after comet impacts from millimetre observations. Nature 373, 592.

Maillard, J.-P., Drossard, P., Bézard, B., De Bergh, D., Lellouch, E., Marten, A., Caldwell, J., Hilico, J.-C., \& Atreya, S. K. 1995 Methane and carbon monoxide infrared emissions observed at the Canada-France-Hawaii Telescope during the collision of comet SL-9 with Jupiter. Geophysical Research Letters 22, 1573-1576.

Nicholson, P. D., Gierasch, P. J., Hayward, T. L., McGhee, C. A., Moersch, J. E., Squyres, S. W., Van Cleve, J., Matthews, K., Neugebauer, G., Shupe, D., WeinBerger, A., Miles, J. W., \& Conrath, B. J. 1995a Palomar observations of the R impact of comet Shoemaker-Levy 9: I. Light curves. Geophysical Research Letters 22, 1613-1616.

Nicholson, P. D., Gierasch, P. J., Hayward, T. L., McGhee, C. A., Moersch, J. E., Squyres, S. W., Van Cleve, J., Matthews, K., Neugebauer, G., Shupe, D., WeinBerger, A., Miles, J. W., \& Conrath, B. J. 1995b Palomar observations of the R impact of comet Shoemaker-Levy 9: II. Spectra Geophysical Research Letters 22, 1617-1620.

Orton, G. AND 57 others 1995a Collision of comet Shoemaker-Levy 9 with Jupiter observed by the NASA Infrared Telescope Facility. Science 267, 1277-1282.

Orton, G., Spitale, J., Friedson, J., Yanamandra-Fisher, P., Baines, K., Hoffmann, W., DAYAl, A., DEUTSCH, L., \& HorA, J. 1995b Spatial variation and time dependence of the temperature structure of impact sites. Paper presented at IAU Colloquium 156: The Collision of Comet P/Shoemaker-Levy 9 and Jupiter, Baltimore, Maryland, 9-12 May.

WANG, Y., Noll, K. S., \& Yelle, R. 1995 Thermal effects from minor constituents in Jupiter's upper atmosphere after SL9. Paper presented at IAU Colloquium 156: The Collision of Comet P/Shoemaker-Levy 9 and Jupiter, Baltimore, Maryland, 9-12 May. 